\title{
artigo
}

Gonçalves, L.X.R.; Ferreira, C.F.S.; Oliveira, M.S.S.; Lira, P.F.; Pereira, D.C.R.; Lopes da Silva, C.R.

Assistência do enfermeiro acerca da gestante com pré-eclâmpsia no pré-natal

\section{Assistência do enfermeiro acerca da gestante com pré-eclâmpsia no pré-natal}

\author{
Assistance of the nurster acceracking the manager with pre-echlampsy in the pre-natal \\ Asistencia del nuestro que acepta el gestro con preclampsia en el pre-natal
}

\begin{abstract}
RESUMO
Objetivou-se analisar na literatura a assistência do enfermeiro acerca da gestante com pré-eclâmpsia no pré-natal. Trata-se de uma revisão sistemática da literatura com metassíntese, que segue o protocolo PRISMA. Foram utilizados como fonte de busca as bases de dados: SciELO, LILACS e BDENF, apresentando 30 documentos. Após critérios de inclusão: artigos disponíveis integralmente, de forma gratuita, publicados entre os anos de 2016 a 2020, estarem escritos em português, inglês e espanhol. E exclusão: os artigos sem ideia central pautada na temática abordada, estudos repetitivos em diferentes bases de dados, dissertações, documentários, monografias, projetos, resumos, relatos e teses, resultando em um total de 7 artigos. Identificou-se que o enfermeiro presta uma assistência adequada, mas insuficiente, necessitando aprofundar nas orientações com essas gestantes sobre sua patologia e buscar estratégias para o seu autocuidado, de modo a diminuir possiveis complicações relacionado a doença. Desta forma, irá possibilitar um atendimento humanizado e centrado no perfil de cada paciente, prevenindo intercorrências futuras ao binômio materno-fetal.
\end{abstract}

DESCRITORES: Enfermagem. Pré-eclâmpsia. Pré-natal.

\section{ABSTRACT}

The objective was to analyze in the literature the care of nurses about pregnant women with prenatal preeclampsia. This is a systematic review of the literature with metasynthesis, which follows the PRISMA protocol. The following databases were used as a search source: SciELO, LILACS and BDENF, presenting 30 documents. After inclusion criteria: articles available in full, free of charge, published between 2016 and 2020, are written in Portuguese, English and Spanish. And exclusion: articles with no central idea based on the theme addressed, repetitive studies in different databases, dissertations, documentaries, monographs, projects, abstracts, reports and theses, resulting in a total of 7 articles. It was identified that the nurse provides adequate but insufficient care, needing to deepen the guidance with these pregnant women about their pathology and seek strategies for their self-care, in order to reduce possible complications related to the disease. In this way, it will enable humanized care centered on the profile of each patient, preventing future complications to the maternal-fetal binomial.

DESCRIPTORS: Nursing. Preeclampsia. Prenatal.

\section{RESUMEN}

El objetivo era analizar en la literatura el cuidado de enfermeras sobre mujeres embarazadas con preeclampsia prenatal. Esta es una revisión sistemática de la literatura con metasíntesis, que sigue el protocolo PRISMA. Las siguientes bases de datos fueron utilizadas como fuente de búsqueda: SciELO, LILACS y BDENF, presentando 30 documentos. Después de los criterios de inclusión: los artículos disponibles en su totalidad, de forma gratuita, publicados entre 2016 y 2020, están escritos en portugués, inglés y español. Y exclusión: artículos sin idea central basados en el tema abordado, estudios repetitivos en diferentes bases de datos, disertaciones, documentales, monografías, proyectos, resúmenes, informes y tesis, dando como resultado un total de 7 artículos. Se determinó que la enfermera proporciona atención adecuada pero insuficiente, necesitando profundizar la orientación con estas mujeres embarazadas sobre su patología y buscar estrategias para su auto-cuidado, con el fin de reducir las posibles complicaciones relacionadas con la enfermedad. De esta manera, permitirá una atención humanizada centrada en el perfil de cada paciente, previniendo futuras complicaciones en el binomio materno-fetal.

DESCRIPTORES: Enfermería. Preeclampsia. Prenatal.

RECEBIDO EM: 15/03/2021 APROVADO EM: 22/06/2021 


\section{Laudilina Xavier Rocha Gonçalves}

Enfermeira. Pós-graduanda em Saúde da Família - FAVENI.

ORCID: 0000-0002-2352-7054

\section{Carolina Francisca da Silva Ferreira}

Enfermeira. Pós-graduanda em Saúde da Família - FAVENI.

ORCID: 0000-0003-1955-0005

\section{Maria do Socorro Santos de Oliveira}

Enfermeira. Especialista em Enfermagem Obstétrica, Docente de Enfermagem - UNIJUAZEIRO.

ORCID: 0000-0001-9392-2378

\section{Petrúcya Frazão Lira}

Enfermeira. Mestre em Ciências da Educação, Especialista em Gestão de Redes, Docente da UNIJUAZEIRO, Coordenadora da Escola Técnica do SUS Dr. Antonio Marchet Callou.

ORCID: 0000-0001-9539-066X

\section{Dayse Christina Rodrigues Pereira}

Enfermeira. Doutora em Ciência da saúde, pelo Centro Universitário Saúde do ABC-FMABC, Diretora da Rede Municipal de Saúde Mental, Pós Doutoranda em Ciência da Saúde pelo centro Universitário Saúde do ABC-FMABC.

ORCID: 0000-0002-5719-3574

\section{Cicero Rafael Lopes da Silva}

Enfermeiro. Especialista em Enfermagem Dermatológica, Pós-graduando em Docência do Ensino Superior - UNIJUAZEIRO e em Gerontologia e saúde do idoso - FAVENI, Docente da graduação em Enfermagem - UNIJUAZEIRO.

ORCID: 0000-0001-8819-5380

\section{INTRODUÇÃO}

A gravidez acontece de forma natural, fisiológica e dinâmica, havendo várias mudanças durante esse período, mesmo que ocorra o cuidado durante o seu percurso, a gestante poderá desenvolver complicações, como as Síndromes Hipertensivas Específicas da Gestação (SHEG) que representa um dos problemas de saúde pública. É caracterizada por um aumento da Pressão Arterial (PA) e pode ter resultados negativos durante $\mathrm{o}$ desfecho gestacional ${ }^{1}$.

A pré-eclâmpsia ( $\mathrm{PE}$ ) é uma doença multissistêmica, se tornando uma das SHEG que mais acomete as gestantes durante a gravidez. Ocorre especificamente após a $20^{a}$ semana de gestação e é detectada através da tríade sintomática: hipertensão, proteinúria e edema. Muitas vezes a doença se desenvolve silenciosamente sem a presença de sintomas sugestivo da pré-eclâmpsia. A PE quando não diagnosticada precocemente pode evoluir para a eclampsia trazendo complicaçóes graves para a gestante e o feto ${ }^{2}$.
É importante ressaltar, que existem alguns fatores de risco que aumentam a probabilidade de uma gestante apresentar pré-eclâmpsia, como por exemplo: histórico da doença em gestações anteriores, hipertensão crônica, doença renal crônica, diabetes mellitus pré-existentes, idade superior a 40 anos, obesidade e a etnia. Apesar da importância do ponto de vista de saúde pública, a causa subjacente a essa condição permanece desconhecida $a^{3-4}$.

Cerca de $2 \%$ a $8 \%$ de todas as gestantes são acometidas pela PE, ou seja, no Brasil, constitui a primeira causa de morte materna, a doença não se associa apenas a elevada taxa de mortalidade, mais dos agravos permanentes que pode trazer ao binômio materno-fetal, levando a uma necessidade maior de cuidados assistenciais ${ }^{4}$.

Segundo a Organização Mundial de Saúde (OMS), o sugerido é de 20 óbitos maternos para cada 100.000 nascidos vivos, o índice de mortalidade materna no estado do Ceará causada pela doença é de 78.5 óbitos para cada 100.000 nascidos vivos. Ou seja, para diminuir essa taxa de mortalidade, visa a importância do pré-natal e atendimento hospitalar de qualidade $^{5-6}$.

Diante de tais considerações, questiona-se: Qual a assistência do enfermeiro acerca da gestante com pré-eclâmpsia no pré-natal?

Assim, o enfermeiro através da prestação de cuidados, deve exercer o papel de acolher, orientar e informar sobre a doença e seus riscos. Sendo de grande importância a gestante conhecer todas as condutas necessárias que deverão ser feitas diante da sua patologia, de tal modo, a colaborar com a assistência prestada e ajudar o profissional na elaboração de estratégias para o seu autocuidado. $\mathrm{O}$ atendimento a essas mulheres dispõe de metas e planos de cuidados, de maneira a implementar a Sistematização da Assistência de Enfermagem (SAE), que enfatiza a assistência do profissional enfermeiro não só como de recursos técnicos, mas da realização do cuidado holístico, ou seja, avaliando a paciente em todas as dimensões.

Deste modo, esse estudo é de total relevância sobre a assistência do enfermeiro na pré-eclâmpsia para a saúde materno- 
-fetal, visto que é fundamental oferecer um acompanhamento singularizado a gestante durante o pré-natal. De forma, a estabelecer um vínculo de confiança entre a paciente e o profissional envolvido para conseguir enfrentar todas as adversidades que poderão surgir durante o período da gravidez, principalmente no contexto do pré-natal de alto risco.

Diante de tais preceitos, a presente pesquisa objetivou analisar na literatura a assistência do enfermeiro acerca da gestante com pré-eclâmpsia no pré-natal.

\section{METODOLOGIA}

Trata-se de uma revisão sistemática de literatura com metassíntese. As revisões sistemáticas têm o propósito de investigar cientificamente, de maneira a dispor de modo conjunto de vários autores, avaliar criteriosamente e em seguida redigir uma síntese dos resultados dos diversos estudos primários. Essa modalidade de pesquisa, é útil para agregar as opiniões de um conjunto de documentos socializados por vários autores sobre determinada temática, podendo apresentar resultados coincidentes ou opostos?

Foi realizada a busca de elaborações cientificas sobre a assistência do enfermei- ro acerca da gestante com pré-eclâmpsia no pré-natal, para alcançar a produção científica, foram utilizadas como fonte de busca a Biblioteca Virtual em Saúde (BVS) e as bases de dados: Scientific Electronic Library Online (SciELO), Literatura Latino-americana e do Caribe em Ciências da Saúde (LILACS) e Base de Dados em Enfermagem (BDENF). A sondagem das obras científicas aconteceu entre os meses de abril a setembro de 2020 .

Sendo utilizados como fonte de pesquisa os descritores em Ciência da Saúde (DeCS): "enfermagem", "pré-eclâmpsia" e "pré-natal" os descritores citados foram associados ao operador booleano AND, da seguinte forma: "enfermagem" AND "pré-eclâmpsia” AND "pré-natal”

Foram incluídos na pesquisa, os estudos que atendiam aos seguintes critérios empregados: artigos disponíveis integralmente de maneira gratuita, publicados entre os anos de 2016 a 2020, estarem escritos em português, inglês e espanhol. Foram excluídos da pesquisa, os artigos sem ideia central pautada na temática abordada, estudos repetitivos em diferentes bases de dados, dissertações, documentários, monografias, projetos, resumos, relatos e teses.

Para a seleção dos dados, foi feita uma leitura criteriosa do conteúdo encontra-

\section{Figura 01: Fluxograma do processo de obtenção dos artigos revisados.}

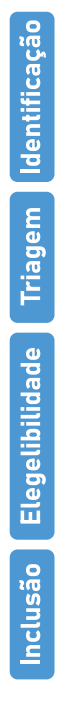

Estudos identificados

através da pesquisa no

banco de dados: $(n=30)$

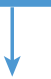

Estudos após remoçăo de duplicatas $(n=25)$

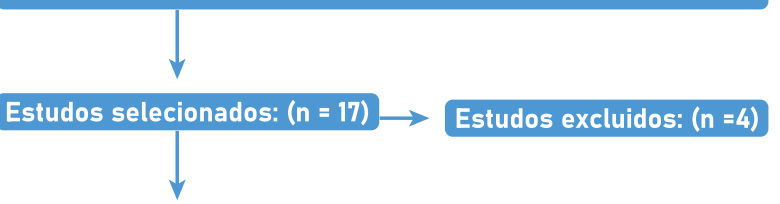

Artigos completos avaliados para elegibilidade $(n=13)$

Artigos completos

excluídos com razão $(n=6)$
Documentos adicionais

identificados por outras fontes $(n=1)$
Estudos incluídos na sintese qualitativa $(n=7)$ do. A princípio, foram lidos os títulos e os resumos de todos os artigos selecionados, assim efetivando a seleção dos conteúdos que atenderam aos critérios de inclusão e descartando os que não faziam parte do mesmo.

No que tange aos aspectos éticos da pesquisa para as revisões, não se faz necessário a submissão ao Comitê de ética e pesquisa com seres humanos. Uma vez que, os artigos utilizados já passaram pela a apreciação ética e encontram-se disponíveis online nas bases de dados. Logo, é possível afirmar que todos os preceitos da resolução do Conselho Nacional de Saúde $n^{\circ} 466 / 12$ foram respeitados.

\section{RESULTADOS}

Foi realizado uma busca inicial na BVS, sendo filtradas as bases de dados LILACS e BDENF com uma obtenção de 24 documentos encontrados. $\mathrm{Na}$ base de dados SciELO, 6. Com uma totalidade de 30 documentos. Após aplicação dos critérios estabelecidos, restaram 13 artigos, os quais foram pré-selecionados. Destes 13 estudos, 6 não estavam disponíveis na integra eletronicamente ou não eram artigos originais. Assim, somente 7 artigos concederam com o objeto de análise do estudo, dos quais, LILACS (04), SciELO (01) e BDENF (02). Com o intuito de demonstrar o processo de busca e escolha dos estudos, utilizou-se um fluxograma baseado no protocolo PRISMA, ilustrado na figura 1.

\section{DISCUSSÃO}

Com base nos estudos incluídos nessa revisão, foi possível identificar que a maioria dos autores estavam em consonância em relação a importância do pré-natal de qualidade frente as mulheres com pré-eclâmpsia, porém, identificou-se que o enfermeiro presta uma assistência adequada, mas insuficiente. Necessitando aprofundar nas orientações com essas gestantes sobre sua patologia e buscar estratégias para o seu autocuidado, de modo a diminuir possíveis complicações materno-fetais relacionado a doença. 
Tabela 1: Distribuição dos artigos selecionados segundo autor, ano, objetivo, abordagem metodológica e principais resultados.

AUTOR/ANO

Abrahão ACM, Santos RFS, Viana SRG, Viana

$\mathrm{SM}, 2020$.

Kahhale S, Francisco RPV, Zugaib M, 2018.

Silva DF, Jesus EG, Peres LC, 2018.

Amorim FCM, Neves ACN, Moreira FS, Oliveira ADS, Nery IS, 2017.

Junior ARF, Filho JTO, Rodrigues MENG, Albuquerque RAS, Siqueira DA, Rocha FAA, 2017

Oliveira GS, Paixão GPN, Fraga CDS, Santos MKR, Santos MA, 2017.

Oliveira KKPA de, Andrade SSC, Silva FMC, Meneses LBA, Leite KNS, Oliveira SHS, 2016.
OBJETIVOS

Identificar a importância da assistência de enfermagem às gestantes com Síndrome Hipertensiva Gestacional.

Descrever a pré-eclâmpsia, e a terapêutica farmacológica anti-hipertensiva na gestação.

Descrever o conhecimento dos profissionais de enfermagem sobre as gestantes com suspeita de DHEG na Unidade Básica de Saúde (UBS).

Descrever as complicações das gestantes com PE.

Conhecer o papel do enfermeiro no atendimento ao pré-natal de alto risco realizado na atenção secundária.

Analisar a assistência de enfermeiros às gestantes com síndrome hipertensiva, em um hospital de baixo risco obstétrico.

Avaliar a assistência de enfermagem prestada à mulher acometida por pré-eclâmpsia.
PRINCIPAIS RESULTADOS

É compromisso da gestante em cuidar de si e de seu concepto, participando ativamente dos programas e consultas durante a gravidez.

O melhor tratamento para pré-eclâmpsia, continua sendo a assistência no pré-natal correto.

O enfermeiro é um dos profissionais mais apto para reconhecer as DHEG e prestar assistência.

Uma assistência pré-natal e puerperal qualificada e humanizada.

A educação em saúde na gestação de alto risco é primordial.

É possível perceber a influência direta da assistência pré-natal nos atendimentos de pacientes que adentram na maternidade.

As gestantes merecem além dos cuidados rotineiros, atenção especial.
Sob esta perspectiva, durante a gestação de alto risco a mulher poderá passar por complicações proveniente da pré-eclâmpsia, onde será necessário a participação ativa do sistema de saúde, principalmente da enfermagem, prestando uma assistência projetada nas necessidades de cada paciente, assim, irá prevenir a morbimortalidade materna e perinatal ${ }^{8}$.

Em estudos realizados, apontaram que mulheres que desenvolveram pré-eclâmpsia devem ser prevenidas de uma eclampsia, ou síndrome de HELLP (hemólise, elevação das enzimas hepáticas, e queda na contagem de plaquetas), ou seja, precisam ser acompanhadas na atenção primária, de modo que esteja complementada com a atenção secundária na ocorrência de complicações. O diagnóstico realizado precocemente facilita a tomada de decisões para prevenção de consequências mais severas relacionada a síndrome $e^{4-9}$.

Em benefícios de fatos referidos, o Ministério da Saúde preconiza o uso de uma abordagem integral a essas mulheres, e a importância do manejo adequado do processo saúde doença. Além disso, é de

\section{Em benefícios de}

fatos referidos,

o Ministério da

Saúde preconiza

o uso de uma

abordagem integral

a essas mulheres, e

a importância do

manejo adequado

do processo saúde

doença. grande relevância seguir as recomendações da Organização Mundial de Saúde (OMS) sobre os protocolos assistenciais, e as informações do Sistema de Informação Sobre Nascidos Vivos (SINASC), assim, irá diminuir o índice de mortalidade e ajudará o profissional em conjunto com a gestante a prevenir a evolução da pré-eclâmpsia-10.

Estudos mostram que o atendimento a gestante na atenção primária é realizado mediante o Sistema Único de Saúde (SUS) que preconiza a importância do pré-natal. Dessa forma, o Ministério da Saúde estabelece que deve ser feito no mínimo seis consultas de pré-natal, onde os profissionais possam identificar mulheres que tenham probabilidade de desenvolverem essa síndrome, e em seguida prestar um acompanhamento em todo o período da gravidez, parto e puerpério ${ }^{11-12}$.

Contudo, autores indagam, que a pré-eclâmpsia representa uma das principais causas de mortalidade materna, se tornando um risco para a saúde pública, podendo desencadear doenças a longo prazo, ou seja, depois do parto, como: doenças car- 


\section{artigo}

Gonçalves, L.X.R.; Ferreira, C.F.S.; Oliveira, M.S.S.; Lira, P.F.; Pereira, D.C.R.; Lopes da Silva, C.R.

Assistência do enfermeiro acerca da gestante com pré-eclâmpsia no pré-natal

diovasculares, hipertensão arterial para a mulher e criança ${ }^{4}$.

E baseando-se neste fator, autores destacam as causas que podem contribuir para a elevada taxa de grávidas que desenvolvem pré-eclâmpsia, estar na falta de capacitação do profissional em realizar o rastreio de mulheres que são fatores de risco para o desenvolvimento da PE, da prestação de uma educação continuada, do fornecimento de informações através da assistência adequa$\mathrm{da}$, o que dificulta a resolutividade de problemas diante da gravidez ${ }^{13}$.

Entretanto, percebe-se que a assistência do enfermeiro no pré-natal é imprescindível, de maneira a prestar um atendimento integral e compartilhado, amparado em teoria disciplinar, por meio da multidisciplinaridade. Então o profissional enfermeiro adjunto de uma equipe preparada e com o mesmo objetivo, irá garantir um cuidado resolutivo e de qualidade ao binômio materno-fetal.

\section{Entretanto,}

percebe-se que

a assistência

do enfermeiro

no pré-natal é imprescindível...

\section{CONCLUSÃO}

Esta pesquisa permitiu analisar a assistência do enfermeiro as gestantes com pré-eclâmpsia, sendo possível perceber que essa atenção é de grande importância para manutenção e preservação de vida da mulher e do feto. Visto que estes profissionais possuem autonomia e conhecimento técnico-científico, que quando adjunto a uma equipe multiprofissional desencadeia um trabalho resolutivo.

Contudo, o enfermeiro deve promover qualidade na sua assistência diante da gestante com pré-eclâmpsia, que esteja direcionada para a promoção da saúde, prevenção de agravos e reabilitação. Desta forma, irá possibilitar um atendimento humanizado e centrado no perfil de cada gestante, prevenindo intercorrências futuras ao binômio materno-fetal..

\section{REFERÊNCIAS}

1. Caires TLG, Souza LMM, Ramos SF, Leite LBV, Matos SCN, Santos MHRS. Revisão integrativa sobre síndrome hipertensiva gestacional: instrumento teórico como norteador para atividade prática de acadêmicos do curso de enfermagem. Expressa extenção, v.25, n.1, p.91-106, 2020. Disponivel em: https://doi.org/10.15210/ ee.v25i1.17247.

2. Ferreira MBG, Silveira CF, Silva SR, Souza DJ, Ruiz MT. Assistência de enfermagem a mulheres com pré-eclâmpsia e/ou eclâmpsia: revisão integrativa. Revista da Escola de Enfermagem USP, v. 50, n.2, p.324-334, 2016. Disponível em: DOI: http://dx.doi.org/10.1590/ S0080-623420160000200020.

3. Santos NAS, Gurgel JAA, Camurça CG. Avaliação dos fatores de risco materno em gestante admitidas com pré-eclâmpsia grave. Revista Medicina UFC, v.56, n.2, p.25-29, 2016. Disponível em: DOI: 10.20513/2447-6595.2016v56n2p25-29.

4. Kahhale S, Francisco RPV, Zugaib M. Pré-eclâmpsia. Revista Medicina (São Paulo), v.97, n.2, p. 226-234,2018. Disponivel em: http:// dx.doi.org/10.11606/issn.1679-9836.v97i2p226-234.

5. Catunda HLO, Mendes IC, Sousa DMN, Damasceno AKC, Oriá MOB. Razão da mortalidade materna no ceará: uma análise epidemiológica. Seminário nacional de pesquisa em enfermagem. 2013. Disponível em: http://www.abeneventos.com.br/anais_senpe/17senpe/pdf/1533co.pdf.

6. Amorim FCM, Neves ACN, Moreira FS, Oliveira ADS, Nery IS. Perfil de gestantes com pré-eclâmpsia. Revista enfermagem UFPE online, v.11, n.4, p.1574-83, 2017. Disponível em: DOl: 10.5205/reuol.976385423-1-SM.1104201703.

7. Maria CBG, Ivan LMR. Revisão Sistemática Da Literatura: Conceituação, Produção e Publicação. Logeion Filosofia da Informação [Internet]. v. 6, n. 1, 2019. Disponivel em: < http://revista.ibict.br/fiinf/ article/view/4835/4188>.

8. Silva DF, Jesus EG, Peres LC. Assistência de enfermagem na unidade básica de saúde na doença hipertensiva específica na gestação. Revista de Enfermagem da Faciplac. v.2, n.2, p.1-11, 2018. Disponivel em: http://revista.faciplac.edu.br/index.php/REFACI/article/ view/575.

9. Abrahão ACM, Santos RFS, Viana SRG, Viana SM. Atuação do enfermeiro a pacientes portadoras de Síndrome Hipertensiva Específica da Gestação. Revista Cientifica da Escola Estadual de Saúde Pública Goiás "Candido Santiago". v.6, n.1, p.51-63, 2020. Disponível em: http://www.revista.esap.go.gov.br/index.php/resap/article/ view/192/192.

10. Oliveira GS, Paixão GPN, Fraga CDS, Santos MKR, Santos MA. Assistência de enfermeiros na síndrome hipertensiva gestacional em hospital de baixo risco obstétrico. Revista Cuidarte, v.8, n.2, p.1561-72, 2017. Disponível em: http://dx.doi.org/10.15649/cuidarte.v8i2.374.

11. Junior ARF, Filho JTO, Rodrigues MENG, Albuquerque RAS, Siqueira DA, Rocha FAA. $O$ enfermeiro no pré-natal de alto risco: papel profissional. Revista Baiana de Saúde Pública. v.41, n.3, p.650-667, 2017. Disponível em: DOI: 10.22278/2318-2660.2017.v41.n3.a2524.

12. Oliveira KKPA de, Andrade SSC, Silva FMC, Meneses LBA, Leite KNS, Oliveira SHS. Assistência de enfermagem a parturientes acometidas por pré-eclâmpsia. Revista de enfermagem da UFPE Online. v.10, n.5, p.1773-80, 2016. Disponivel em: DOl: 10.5205/reuol.900378704-1-SM.1005201625.

13. Gonzaga ICA, Santos SLD, Silva ARV, Campelo V. Atenção pré-natal e fatores de risco associados à prematuridade e baixo peso ao nascer em capital do nordeste brasileiro. Ciência e saúde coletiva. v.21, n.6, p.1965-1974, 2016. Disponível em: DOl: 10.1590/141381232015216.06162015. 